Open Access

\title{
The reliability and minimal detectable change of Timed Up and Go test in individuals with grade 1 - 3 knee osteoarthritis
}

\author{
Ahmad Alghadir ${ }^{1}$, Shahnawaz Anwer ${ }^{1,2^{*}}$ and Jean-Michel Brismée ${ }^{3}$
}

\begin{abstract}
Background: The Timed Up and Go (TUG) test is quick and easy tests to assess patients' functional mobility. However, its reliability in individuals with knee osteoarthritis $(\mathrm{OA})$ has not been well established. The aims of this study were to determine the reliability and minimal detectable change of the TUG test in individuals with doubtful to moderate (Grade 1-3) knee OA.
\end{abstract}

Methods: Sixty-five subjects (25 male, 40 female), aged 45-70 years, with knee OA participated. Inter-rater reliability was assessed using two observers at different times of the same day in an alternating order. Intra-rater reliability was assessed on two consecutive visits with a 2-day interval. The standard error of measurement (SEM) and the minimum detectable change (MDC) were calculated to determine statistically meaningful changes.

Results: Intra-rater and inter-rater reliability were 0.97 (95\% confidence interval [Cl], $0.95-0.98$ ) and 0.96 (95\% confidence interval $[\mathrm{Cl}], 0.94-0.97$ ), respectively. The MDC, based on measurements by a single rater and between raters, was 1.10 and 1.14 seconds, respectively.

Conclusions: The TUG is a reliable test with adequate MDC for clinical use in individuals with doubtful to moderate knee OA.

\section{Background}

Knee osteoarthritis (OA) is a common musculoskeletal disorder affecting the functional mobility of older individuals $[1,2]$. The evidence of radiographic knee OA has been reported to be $53.3 \%$ in male and $60.9 \%$ in female adults aged $30-93$ years in the Middle East [3]. Knee $\mathrm{OA}$ is likely to become the eighth most important global cause of disability in men and the fourth most important in women [4]. The presence of knee pain, decreased functional mobility, stiffness and reduced quadriceps strength has been associated with knee OA and may lead to physical disability [5-7]. Because a major aim of rehabilitation programs for knee $\mathrm{OA}$ is to optimize patients' functional mobility to carry out their activities of daily living (ADLs), therapist require a valid and reliable

\footnotetext{
* Correspondence: anwer_shahnawazphysio@rediffmail.com

'Rehabilitation Research Chair, College of Applied Medical Sciences, King Saud University, Riyadh, Saudi Arabia

${ }^{2}$ Dr. D. Y. Patil College of Physiotherapy, Dr. D. Y. Patil Vidyapeeth, Pune, India Full list of author information is available at the end of the article
}

tool to assess patients' functional mobility at baseline and post intervention.

The Timed Up and Go (TUG) test is one of the simple and quick tests to assess patients' functional mobility. Podsiadlo and Richardson [8] recorded the time taken to complete the TUG in a group of frail elderly subjects with stroke, Parkinson's disease, arthritis, cerebellar disorders, or general deconditioning. In addition to excellent reliability (ICC 0.99), the TUG scores shown moderate correlation with the Barthel Index $(r-0.51)$, gait speed $(r-0.55)$, and the Berg Balance Scale score $(r-0.72)$ [8]. Several other studies reported good test-retest reliability of TUG test in specific subject populations, including communitydwelling older adults $[9,10]$, individuals with Parkinson's disease $[11,12]$, and unilateral lower-limb amputation [13]. Previous research suggested that the TUG test had the capacity, in community dwelling people, to predict the patient's ability to go outside alone safely and to function in other settings [8]. Yeung et al. [14] investigated the test - retest reliability of TUG test in a group of patients admitted in inpatient orthopaedic 
ward (most of them had either total hip or total knee arthroplasty surgery). They reported moderate test - retest reliability (ICC 0.80) and concluded that the TUG test was reliable and valid to assess group changes of patients in orthopaedic rehabilitation wards.

Recently, the Osteoarthritis Research Society International (OARSI) recommended a set of five performancebased tests of physical function, including the TUG test in individuals diagnosed with hip or knee OA [15]. The authors recommended TUG test because it demonstrated good measurement properties in people with $\mathrm{OA}$ and other populations [16-20]. In addition, Dobson et al. [21] conducted a systematic review on the measurement properties of performance-based measures to assess physical function in hip and knee OA. They reported that sit to stand tests with the best measurement evidence included the TUG test and the 30-second chair stand test for hip/ knee OA. In a previous study, Norén et al. [22] investigated the applicability and reliability of some balance assessment methods, including the TUG test, in individuals with peripheral arthritis. They reported that the individuals with severe disability were generally able to perform the TUG test.

Although Kennedy et al. [16] investigated measurement properties of four performance measures including TUG test in patients with advanced OA undergoing total hip or knee arthroplasty, no study to date has estimated the reliability and minimal detectable change of TUG test in a population with doubtful to moderate (Grade 1-3) knee OA. Hence, the purpose of this study was to estimate the reliability and MDC of TUG test in individuals with doubtful to moderate knee OA.

\section{Methods}

\section{Participants and criteria}

All patients diagnosed with knee OA (unilateral or bilateral) by the Physician as per the American College of Rheumatology (ACR) [23] who were referred for outpatient physiotherapy were invited to participate after explanation of the study. Subjects agreeing to participate then signed written consent. Both male and female in the age range of $45-70$ years with pain in and around the knee and radiological evidence of primary grade $1-3$ knee OA on the Kellgren and Lawrence scale [24] were included. Subjects with grade 4 knee OA as per Kellgren and Lawrence scale were excluded as well as subjects with any central or peripheral nervous system involvement, a history of a systemic arthritic condition or of knee surgery to either knee within the past three months. The Kellgren and Lawrence scale classifies OA into four grades as follows: Grade 0 indicates no radiographic findings of $\mathrm{OA}$; Grade 1 indicates minimal osteophytes of doubtful clinical significance; Grade 2 indicates definite osteophytes with unaffected joint space; Grade 3 indicates definite osteophytes with moderate joint space narrowing; and Grade 4 indicates definite osteophytes with severe joint space narrowing and subchondral sclerosis [24]. The Institutional Research Ethics Committee of the Rehabilitation Research Chair, King Saud University, Riyadh, Saudi Arabia, approved the study.

\section{Procedures of data collection}

The study participants' age, sex, height, weight, body mass index (BMI), pain, function, and grade of knee OA were recorded. Pain intensity and knee function were measured using the numerical rating scale (NRS) and the reduced Western Ontario and McMaster Universities Osteoarthritis (WOMAC) index, respectively. The NRS consists of an 11 point horizontal scale from 0 to 10 , with 0 meaning no pain at all and 10 describing the worst pain ever. It is a reliable and valid instrument for assessing musculoskeletal and arthritic pain [25, 26]. The 5-point likert version of the reduced WOMAC index was used to assess knee function [27, 28].

Two licensed physiotherapists with more than 8 years of clinical practice and experience in the TUG test administration performed inter-rater reliability testing at different times of the same day in an alternating order. Both clinicians were trained in the administration of TUG test for the purpose of standardization of the instructions. For intra-rater reliability testing, the same physiotherapists performed the TUG on two consecutive visits with 2-day interval. The TUG test was administered by one examiner in a quiet area [8]. Subjects were instructed to stand up from the chair, walk 3 meters comfortably and safely, come back and sit back in the chair. The time taken to complete this task was measured with a stopwatch timed to the nearest $1 / 100$ seconds. A practice trial was given and then followed by 2 recorded trials. An average of the 2 recorded trials was used in data analysis.

\section{Statistical analysis}

Descriptive statistics were used to analyze subjects' demographic characteristics and baseline measurements. To determine inter- and intra-rater reliability of TUG measurements between the 2 testing sessions, intraclass correlation coefficients $\left(\mathrm{ICC}_{2,1}\right)$ were used. The BlandAltman plot method was then used to assess the agreement between two readings. The plot comprises of the average of the paired values from two readings on the $\mathrm{x}$-axis and the difference of each set of readings on the $y$-axis. Data were visually interpreted to determine the consistency of two scores. The standard error of measurement (SEM) and the minimum detectable change (MDC) were calculated using the results of the reliability analyses. The SEM is the commonest statistic reported in previous studies for assessing statistically meaningful changes of a health outcome [29, 30]. MDC was calculated as $1.96 \sqrt{2}$ (SEM) [31]. All statistical analyses were 
performed with SPSS for Windows version 22 (Statistical package for Social Sciences, IBM Inc.), and the significance level was set at 0.05 .

\section{Results}

Of the 80 subjects recruited, 15 (Grade 4 OA, $\mathrm{n}=8$; age $>80$ years, $\mathrm{n}=7$; men 5 , women 10 ) were excluded due to not meeting inclusion criteria. Table 1 details the participants' characteristics. The mean age and standard deviation of the male and female participants were 54.3 (10.1) and 51.4 (9.7) years, respectively. Thirty-nine participants $(40 \%)$ had unilateral while the others $(60 \%)$ had bilateral knee OA. Thirty-eight participants had grade 1, 12 grade 2, and 15 grade 3 knee OA as per Kellgren and Lawrence grading system. Table 2 details the baseline scores of TUG, NRS, and WOMAC.

\section{Intra- and inter-rater reliability}

The TUG test for all the participants showed excellent intra- and inter-rater reliability (ICC .97 and .96 , respectively) (Table 3). The Bland-Altman limits of agreement depicted in Figs. 1 and 2 showed a reasonable agreement between the 2 raters (inter-rater) and good agreement between two readings (intra-rater) when differences

Table 1 Participant's characteristics

\begin{tabular}{ll}
\hline Gender, No. (\%) & \\
Male & $25(38.5)$ \\
Female & $40(61.5)$ \\
Age, years & \\
Mean (SD) & $54.87(9.87)$ \\
Minimum - maximum & $45-70$ \\
Height, meter (m) & \\
Mean (SD) & $1.69(0.06)$ \\
Minimum - maximum & $1.56-1.86$ \\
Weight, Kg. & \\
Mean (SD) & $83.15(12.91)$ \\
Minimum - maximum & $56-112$ \\
BMI, Kg./m ${ }^{2}$ & \\
Mean (SD) & $29.04(3.72)$ \\
Minimum - maximum & $21.38-38.75$ \\
KL rating score, no. (\%) & \\
Grade 1 & $38(58.5)$ \\
Grade 2 & $12(18.5)$ \\
Grade 3 & $15(23)$ \\
Uimb involvement, no. (\%) & \\
Bilateral & $39(60)$ \\
\hline
\end{tabular}

BMI Body mass index, $K / L$ Kellgren and Lawrence scale
Table 2 Descriptive statistics of baseline scores

\begin{tabular}{ll}
\hline TUG score (Seconds), All participants & \\
Mean (SD) & $10.88(3.62)$ \\
Minimum - maximum & $6.40-19.90$ \\
TUG score (Seconds), Male & $10.18(2.95)$ \\
Mean (SD) & $7.05-18.76$ \\
Minimum - maximum & \\
TUG score (Seconds), Female & $11.32(3.96)$ \\
Mean (SD) & $6.40-19.90$ \\
Minimum - maximum & \\
TUG score (Seconds), Doubtful knee OA (Grade 1) & $8.46(0.79)$ \\
Mean (SD) & $6.40-9.85$ \\
Minimum - maximum & \\
TUG score (Seconds), Definite knee OA (Grade 2/3) & $14.29(3.28)$ \\
Mean (SD) & $10-19.90$ \\
Minimum - maximum & \\
NRS (0-10) & $5.00(2.17)$ \\
Mean (SD) & $1-9$ \\
Minimum - maximum & \\
WOMAC total score (0-48) & 16.05 (8.91) \\
Mean (SD) & 35 \\
Minimum - maximum & \\
\hline
\end{tabular}

OA Osteoarthritis, TUG Timed up and go test, NRS Numerical rating scale, WOMAC Western Ontario and McMaster Universities Osteoarthritis index ${ }^{\text {a} G r a d i n g ~ a s ~ p e r ~ K e l l g r e n ~ a n d ~ L a w r e n c e ~ s c a l e ~[24] ~}$

between the two readings were plotted against the mean of two readings for all scores. Table 4 shows gender- and grade (OA severity)-wise intra- and inter-rater reliability of the TUG test. The TUG test for male participants showed excellent intra- and inter-rater reliability (ICC .98 and .97 , respectively). The TUG test for female participants showed excellent intra- and inter-rater reliability (ICC .98). The TUG test for doubtful knee OA (Grade 1) showed good intra- and inter-rater reliability (ICC .73 and .71, respectively). The TUG test for definite knee OA (Grade 2-3) showed excellent intra- and inter-rater reliability (ICC .97 and .97 , respectively).

\section{Measurement error and minimum detectable change}

The SEM values were 0.17 seconds and 0.16 seconds, based on repeated measurements for inter- and intrarater, respectively. The MDCs based on the SEM for inter- and intra-rater were 1.14 and 1.10 seconds, respectively (Table 3 ).

\section{Discussion}

Recently, the Osteoarthritis Research Society International (OARSI) recommended the use of the TUG test as a performance-based test of physical function in individuals diagnosed with hip or knee OA [15]. In 
Table 3 Inter- and Intra-rater reliability of Timed Up and Go test - ICCS, SEM, MDC and Bland and Altman tests

\begin{tabular}{|c|c|c|c|c|c|c|c|}
\hline & \multicolumn{3}{|l|}{ ICC, SEM and MDC } & \multicolumn{4}{|c|}{ Bland and Altman test } \\
\hline & ICC (95 \% Cl) & SEM & $\mathrm{MDC}$ & đ & $95 \% \mathrm{Cl}$ for đ & $S D_{\text {diff }}$ & $95 \%$ limit of agreement \\
\hline Inter-rater reliability & $0.96(0.94-0.97)$ & 0.17 & 1.14 & -.045 & $-.24-.15$ & .80 & $-1.61-1.52$ \\
\hline Intra-rater reliability & $0.97(0.95-0.98)$ & 0.16 & 1.10 & -.115 & $-.34-.11$ & .94 & $-1.95-1.72$ \\
\hline
\end{tabular}

ICC intraclass correlation coefficients, SEM standard error of measurement, MDC minimal detectable change, đ mean difference, $95 \%$ CI for $₫ 95 \%$ confidence interval for the mean difference, $S D_{\text {diff }}$ standard deviation of the differences

addition, Dobson et al. [21] reported that the TUG test displayed best measurement evidence among sit to stand tests for hip/knee OA. This is the first study to estimate the reliability and MDC of TUG test in individuals with doubtful to definite radiographic knee OA (Kellgren and Lawrence grades 1-3). The results indicated that the TUG test is sufficiently reliable and sensitive to detect small clinical changes, with psychometric properties in agreement with those reported in most studies on the elderly population (ICC range, $0.92-$ $0.99)[8,10-13]$. Both men and women displayed excellent reliability (ICC range, $0.97-0.98$ ). Similarly, Norén et al. [22] reported excellent reliability $(r=0.97)$ of TUG test in individuals with peripheral arthritis. However, the subjects in their study were primarily individuals with rheumatoid arthritis.

In the present study, the participants with doubtful and definite knee OA had good and excellent reliability (ICC .71 and .97, respectively). Likewise, Kennedy et al. [16] reported moderate to good reliability of the
TUG in patients with advanced OA undergoing total hip or knee arthroplasty. Although the characteristics of the participants included in the Kennedy et al. study [16] and in the present study were different in regard to the severity of the OA condition, both studies found good reliability, thereby indicating the value of the TUG for populations with various levels of OA severity. Patients with advanced knee OA would be expected to display increased performance variability, thus reducing the reliability of repeated measurements.

The mean TUG score obtained in among individuals with knee OA $(10.9 \pm 3.6 \mathrm{~s})$ was lower than that of older adults who functioned independently $(8.1 \pm 1.3 \mathrm{~s})$ [32]. The female participants had lower TUG score than male participants (11.3 s versus $10.2 \mathrm{~s})$. Similarly, the participants with definite knee OA had lower TUG score than doubtful knee OA (14.3 s versus $8.5 \mathrm{~s}$ ). The presence of knee pain and quadriceps muscle weakness is associated with knee OA [5-7], which could explain the lower

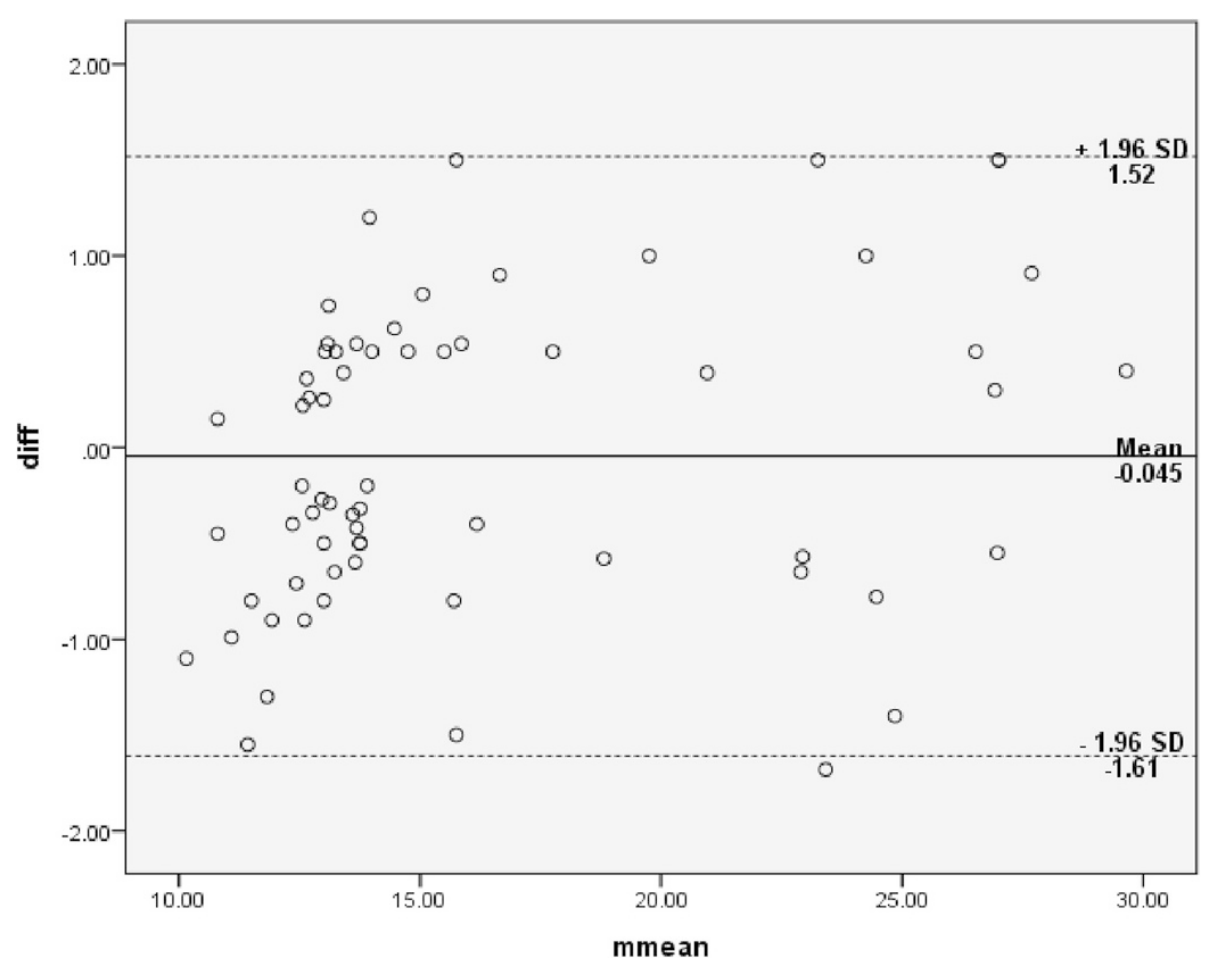

Fig. 1 Bland-Altman plot of difference of two raters with mean (bias) and \pm standard deviation (SD) of differences of two raters 


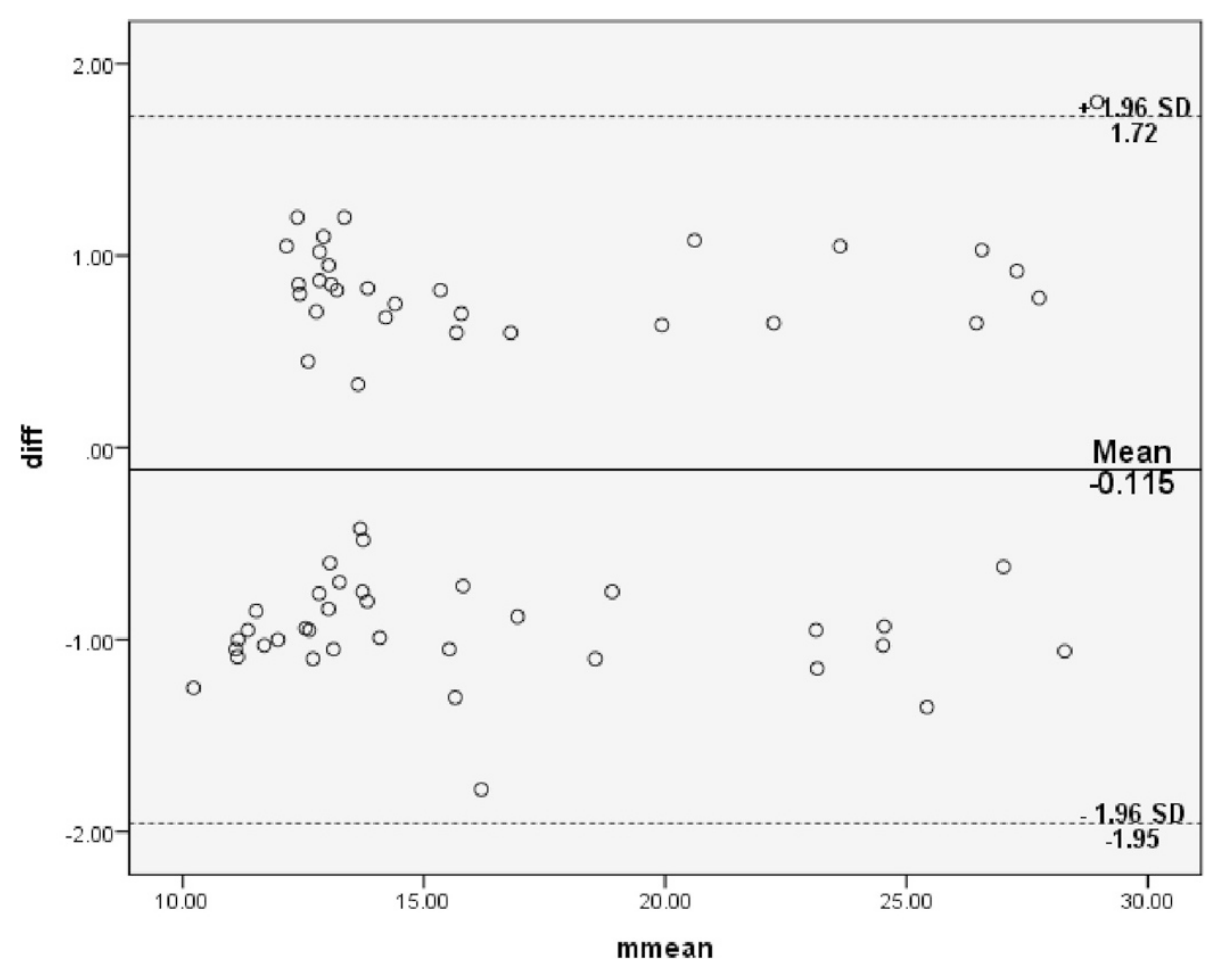

Fig. 2 Bland-Altman plot of difference of two readings for the same rater with mean (bias) and \pm standard deviation (SD) of differences of two readings

TUG score in subjects with knee OA as compared to healthy older adults.

The values of SEM and the MDC were used to calculate measurement error. It is the speculative difference between an observed score on any specific assessment and the actual score for the method [33]. The value of the SEM and MDC provides a threshold for interpreting the TUG over time. The difference between the MDC values based on the SEM for one rater $(1.10 \mathrm{~s})$ and 2 raters $(1.14 \mathrm{~s})$ was small $(0.04 \mathrm{~s})$; therefore, we suggest choosing $1 \mathrm{MDC}$ value to avoid the use of multiple values. Hence, we chose to use the larger MDC (that based on the SEM between two raters). Using this criterion, when the TUG score changes by over $1.14 \mathrm{~s}$, one can be reasonably sure that a true change has occurred, and not just measurement error or noise. Knowledge of the MDC is important to compare the changes in performance-based measures of function in individuals with knee OA. However, Kennedy et al. [16] reported higher SEM (1.07 s) and MDC (2.49 s) as compared to the present study. This may be due to the difference in the participants' characteristics. Participants in their study were individuals with advanced OA undergoing total hip or knee arthroplasty, while individuals with grade 4 knee OA were excluded from this study. In addition, Norén et al. [22] reported higher SEM (1 s) for individuals with peripheral arthritis with mild to severe disability.

The radiographic knee OA severity for various grades is considered debatable in the literature and the use of radiographs for diagnosing knee $\mathrm{OA}$ in person with knee pain in primary care is considered inappropriate [34-36]. Hence, we opted to keep the phrase "doubtful to moderate knee osteoarthritis" for grade 1-3.

Generalization of our results should be limited to the individuals with knee OA with a radiographic grade up to 3 as per Kellgren and Lawrence scale [24]. The sample did

Table 4 Inter- and Intra-rater reliability of Timed Up and Go test: gender- and grade (OA severity)-wise

\begin{tabular}{|c|c|c|c|c|}
\hline & $\begin{array}{l}\text { Male ICC } \\
(95 \% \mathrm{Cl}) \mathrm{N}=25\end{array}$ & $\begin{array}{l}\text { Female ICC } \\
(95 \% \mathrm{CI}) \mathrm{N}=40\end{array}$ & $\begin{array}{l}\text { a Doubtful OA (Grade 1) ICC } \\
(95 \% \text { CI) N=38 }\end{array}$ & $\begin{array}{l}\text { aDefinite OA (Grade 2-3) ICC } \\
(95 \% \text { Cl) N=27 }\end{array}$ \\
\hline Inter-rater reliability & $0.97(0.93-0.98)$ & $0.98(0.97-0.99)$ & $0.71(0.44-0.85)$ & $0.97(0.94-0.98)$ \\
\hline Intra-rater reliability & $0.98(0.95-0.99)$ & $0.98(0.97-0.99)$ & $0.73(0.48-0.85)$ & $0.97(0.95-0.99)$ \\
\hline
\end{tabular}


not include individuals with grade 4 knee OA. The presence of grade 4 knee OA would be expected to increase variability of performance, thus reducing the reliability of repeated measurements. In addition, inclusion of healthy control group could have improved the validity. Despite these limitations, we believe that our study provides estimates of reliability and MDC of TUG scores in individuals with doubtful to moderate knee OA, warranting replication by clinicians in other countries using larger samples of subjects. It would be interesting for future studies to examine the effect of treatment on TUG scores, pain and functional mobility in individuals with knee OA.

\section{Conclusions}

The intra- and inter-rater reliability of the TUG measurements were good to excellent with adequate MDC for clinical use in individuals with doubtful to moderate (Grade 1-3) knee OA. Further study is warranted to validate the TUG test as a single measure of physical function of individuals with knee OA.

\section{Abbreviations}

TUG: Timed Up and Go; MDC: Minimal detectable change; OA: Osteoarthritis; NRS: Numerical rating scale; WOMAC: Western Ontario and McMaster Universities Osteoarthritis Index.

\section{Competing interests}

The authors declare that they have no competing interests.

\section{Authors' contributions}

SA: Corresponding author, participated in the design of the study, participated in the data collection, drafted the manuscript and finished the manuscript. AA: participated in the design of the study and revised the manuscript critically. JMB: participated in the design of the study and revised the manuscript critically. All authors read and approved the final manuscript.

\section{Acknowledgments}

The Project was full financially supported by King Saud University, through Vice Deanship of Research Chairs, Rehabilitation Research Chair.

\section{Funding}

Rehabilitation Research Chair, College of Applied Medical Sciences, King Saud University.

\section{Author details \\ ${ }^{1}$ Rehabilitation Research Chair, College of Applied Medical Sciences, King Saud University, Riyadh, Saudi Arabia. ${ }^{2}$ Dr. D. Y. Patil College of Physiotherapy, Dr. D. Y. Patil Vidyapeeth, Pune, India. ${ }^{3}$ Center for Rehabilitation Research, Texas Tech University Health Sciences Center, Lubbock, Texas, USA.}

Received: 3 February 2015 Accepted: 15 July 2015

Published online: 30 July 2015

\section{References}

1. Helmick CG, Felson DT, Lawrence RC, Gabriel S, Hirsch R, Kwoh CK, et al. Estimates of the prevalence of arthritis and other rheumatic conditions in the United States. Part I Arthritis Rheum. 2008;58(1):15-25.

2. Hootman JM, Helmick CG. Projections of US prevalence of arthritis and associated activity limitations. Arthritis Rheum. 2006;54(1):226-9.

3. Al-Arfaj A, Al-Boukai AA. Prevalence of radiographic knee osteoarthritis in Saudi Arabia. Clin Rheumatol. 2002;21(2):142-5.

4. Murray CJL, Lopez AD. The global burden of disease. Geneva, Switzerland: World Health Organization; 1997.
5. Hurley MV, Scott DL, Rees J, Newham DJ. Sensorimotor changes and functional performance in patients with knee osteoarthritis. Ann Rheum Dis. 1997;56(11):641-8.

6. McAlindon TE, Cooper C, Kirwan JR, Dieppe PA. Determinants of disability in osteoarthritis of the knee. Ann Rheum Dis. 1993;52(4):258-62.

7. Slemenda C, Brandt KD, Heilman DK, Mazzuca S, Braunstein EM, Katz BP, et al. Quadriceps weakness and osteoarthritis of the knee. Ann Intern Med. 1997;127(2):97-104.

8. Podsiadlo D, Richardson S. The timed "Up \& Go": a test of basic functional mobility for frail elderly persons. J Am Geriatr Soc. 1991;39(2):142-8.

9. Hughes C, Osman C, Woods AK. Relationship among performance on stair ambulation, Functional Reach, and Timed Up and Go tests in older adults. Issues on Aging. 1998;21:18-22.

10. Shumway-Cook A, Brauer S, Woollacott M. Predicting the probability for falls in community-dwelling older adults using the Timed Up \& Go Test. Phys Ther. 2000;80(9):896-903.

11. Morris $\mathrm{S}$, Morris ME, lansek R. Reliability of measurements obtained with the Timed "Up \& Go" Test in people with Parkinson disease. Phys Ther. 2001;81(2):810-8.

12. Thompson M, Medley A. Performance of individuals with Parkinson's disease on the Timed Up \& Go. Neurol Rep. 1998;22:16-21.

13. Schoppen T, Boonstra A, Groothoff JW, de Vries J, Goeken LN, Eisma WH. The Timed "Up \& Go" test: reliability and validity in persons with unilateral lower limb amputation. Arch Phys Med Rehabil. 1999;80:825-8.

14. Yeung TSM, Wessel J, Stratford P, Macdermid J. The Timed Up and Go Test for Use on an Inpatient Orthopaedic Rehabilitation Ward. JOSPT. 2008;38(7):410-7.

15. Dobson F, Hinman RS, Roos EM, Abbott JH, Stratford P, Davis AM. Bet al: OARSI recommended performance-based tests to assess physical function in people diagnosed with hip or knee osteoarthritis. Osteoarthritis Cartilage. 2013;21(8):1042-52

16. Kennedy DM, Stratford PW, Wessel J, Gollish JD, Penney D. Assessing stability and change of four performance measures: a longitudinal study evaluating outcome following total hip and knee arthroplasty. BMC Musculoskelet Disord. 2005;6:3.

17. Wright AA, Cook CE, Baxter GD, Dockerty JD, Abbott JH. A comparison of 3 methodological approaches to defining major clinically important improvement of 4 performance measures in patients with hip osteoarthritis. J Orthop Sports Phys Ther. 2011:41:319-27.

18. French HP, Fitzpatrick M, FitzGerald O. Responsiveness of physical function outcomes following physiotherapy intervention for osteoarthritis of the knee: an outcome comparison study. Physiotherapy. 2011;97:302-8.

19. Mizner RL, Petterson SC, Clements KE, Zeni Jr JA, Irrgang JJ, Snyder-Mackler L. Measuring functional improvement after total knee arthroplasty requires both performance-based and patient-report assessments. A longitudinal analysis of outcomes. J Arthroplasty. 2011;26:728-37.

20. Parent $\mathrm{E}$, Moffet $\mathrm{H}$. Comparative responsiveness of locomotor tests and questionnaires used to follow early recovery after total knee arthroplasty. Arch Phys Med Rehabil. 2002;83:70-80.

21. Dobson F, Hinman RS, Hall M, Terwee CB, Roos EM, Bennell KL. Measurement properties of performance-based measures to assess physical function in hip and knee osteoarthritis: a systematic review. Osteoarthritis Cartilage. 2012;20(12):1548-62.

22. Norén AM, Bogren U, Bolin J, Stenström C. Balance assessment in patients with peripheral arthritis: applicability and reliability of some clinical assessments. Physiother Res Int. 2001;6(4):193-204.

23. Altman R, Asch E, Bloch D, Bole G, Borenstein D, Brandt $K$, et al. Development of criteria for the classification and reporting of osteoarthritis. Classification of osteoarthritis of the knee. Diagnostic and Therapeutic Criteria Committee of the American Rheumatism Association. Arthritis Rheum. 1986;29(8):1039-49.

24. Kellgren J, Lawrence J. Radiological assessment of osteoarthrosis. Ann Rheum Dis. 1957;16:494-502.

25. Gallasch $\mathrm{CH}$, Alexandre NM. The measurement of musculoskeletal pain intensity: a comparison of four methods. Rev Gaucha Enferm. 2007;28:260-5.

26. Ferraz MB, Quaresma MR, Aquino LR, Atra E, Tugwell P, Goldsmith CH. Reliability of pain scales in the assessment of literate and illiterate patients with rheumatoid arthritis. J Rheumatol. 1990;17:1022-4.

27. White House SL, Lingard LA, Katz JN, Learmonth ID. Developmental and testing of a reduced WOMAC function scale. JBJS. 2003;85:706-11. 
28. Bellamy N, Buchanan WW, Goldsmith CH, Campbell J, Stitt PW Validation study of WOMAC: a health status instrument for measuring clinically important patient relevant outcomes to antirheumatic drug therapy in patients with osteoarthritis of the hip or knee. J Rheumatol. 1988;15:1833-40.

29. Lydick E, Epstein RS. Interpretation of quality of life changes. Qual Life Res. 1993;2:221-6.

30. Wyrwich KW, Wolinsky FD. Identifying meaningful intra-individual change standards for health-related quality of life measures. J Eval Clin Pract. 2000;6:39-49.

31. Portney $L G$, Watkins MP. Foundations of clinical research: applications to practice. Stamford: Appleton \& Lange; 1993.

32. Giladi N, Herman T, Reider-Groswasser II, Gurevich T, Hausdorff JM. Clinical characteristics of elderly patients with a cautious gait of unknown origin. J Neurol. 2005:252:300-6.

33. Joint Committee on Standards for Educational and Psychological Testing of the American Educational Research Association, American Psychological Association. National Council on Measurement in Education. Standards for educational and psychological testing. Washington (DC): American Educational Research Association; 2002.

34. Bedson J, Jordan K, Croft P. How do GPs use x rays to manage chronic knee pain in the elderly? A case study. Ann Rheum Dis. 2003;62:450-4.

35. Royal College of Radiologists. Making the best use of a Department of Clinical Radiology. Guidelines for doctors. Fourth editionth ed. London: Royal College of Radiologists; 1998.

36. Dutch Orthopaedic Society. Guideline diagnostics and management of hip and knee osteoarthritis [Richtlijn diagnostiek en behandeling van heup-en knieartrose. Nederlandse Orthopaedische Vereniging]. 2007.

\section{Submit your next manuscript to BioMed Central and take full advantage of:}

- Convenient online submission

- Thorough peer review

- No space constraints or color figure charges

- Immediate publication on acceptance

- Inclusion in PubMed, CAS, Scopus and Google Scholar

- Research which is freely available for redistribution 